\title{
Quelles ressources interactionnelles pour la conception de jeux épistémiques numériques?
}

\section{Interactional resources for the design of digital epistemic games}

\author{
Vasiliki Markaki-Lothe ${ }^{1}$ a , Erica de Vries $^{1}$ et Kristine Lund ${ }^{2}$ \\ ${ }^{1}$ Laboratoire LaRAC (EA 602), Université Grenoble Alpes, France \\ ${ }^{2}$ Laboratoire ICAR (UMR 5191), CNRS / École Normale Supérieure de Lyon, France
}

\begin{abstract}
Résumé. L'ouverture actuelle des jeux de formation vers des environnements hybrides (numériques et non numériques) et des objectifs d'apprentissages plus larges complexifie la nature des interactions entre les acteurs durant les différentes phases de la conception d'un tel jeu et interroge les pratiques mobilisées. Notre étude s'attache à décrire les ressources interactionnelles mobilisées lors de la conception de jeux épistémiques numériques. En nous appuyant sur des enregistrements vidéo, nous nous intéressons à la manière dont les pratiques des participants, au fil des différentes phases de la conception d'un jeu, configurent les interactions. Au-delà de l'intérêt théorique, la problématisation des ressources mobilisées est nécessaire à une meilleure compréhension des mécanismes mis en œuvre pour la production d'un jeu et pour l'apprentissage des joueurs. Elle permet ainsi un éclairage sur les conditions qui pourraient avoir un effet sur l'efficacité de ce type de dispositifs.
\end{abstract}

\begin{abstract}
The current interest for video training games that aim to help learners to cope with ill-structured problems questions the kind of interactions and the kind of interactional resources that participants mobilize through consecutive steps within game design. Our study aims to describe several of those interactional resources. Based on video recordings, we look at how participants accomplish their activities and shape interaction within this specific context. Beyond the theoretical interest, the problematization of the interactional resources for design is required in order to ensure better understanding of the interactional mechanisms supporting efficient learning and the efficient design of an epistemic game.
\end{abstract}

\footnotetext{
a Auteure de correspondance : vasiliki.markaki-lothe@univ-grenoble-alpes.fr
} 


\section{Introduction}

La question des ressources effectivement mobilisées dans le domaine des jeux de formation, qu'ils soient numériques ou pas, est au centre des préoccupations à la fois des concepteurs, de ceux qui les utilisent pour former et de ceux qui les évaluent. Dans la littérature, la notion de «ressource » est polysémique. Elle peut par exemple désigner une réalité matérielle, physique et/ou psychique comme des objets, des supports de nature variée, des personnes et des compétences (voir par exemple les travaux de Guichon, 2006 sur la conception multimédia adressés à des enseignants et ceux de Le Boterf, 2011 sur l'évaluation des compétences adressés notamment à des professionnels du management et des ressources humaines). Elle peut se référer à des normes institutionnelles et des pratiques standardisées comme les contrats, les protocoles, les manuels (voir Gueudet et Truche, 2010 sur le travail documentaire des professeurs de mathématiques). Elle peut encore s'étendre dans le domaine de l'activité et considérer les pratiques et leurs modalités de réalisation comme ressource dans ce qu'elles permettent et limitent dans les actions entreprises (voir Filliettaz, 2009 pour une introduction en français de cette approche). C'est cette troisième acception comme entrée analytique, à partir des analyses des interactions, qui nous intéresse ici. Une des difficultés consiste alors à identifier les activités constitutives d'une démarche plus globale comme celle de la conception d'un jeu et à différencier les différentes pratiques qui les constituent ainsi que les ingrédients qui composent ces pratiques.

Dans le domaine des jeux de formation, concevoir, jouer et étudier un jeu représentent trois activités différentes qui relèvent de processus avec des acteurs et des temporalités distincts. Plusieurs travaux, ayant démontré l'intérêt d'entremêler ces processus et estomper les frontières entre les environnements matériels de ces activités lors de la phase de la conception (Bainbridge, 2004), nous invitent à reconsidérer les distinctions entre ces trois activités à la lumière de ce qui les implémente, à savoir les pratiques réelles des acteurs impliqués. Avant de poursuivre, il est important d'apporter quelques éclairages sur l'évolution de la manière de considérer ces distinctions dans le domaine de la conception des jeux de formation et sur les types de questions que cette évolution soulève dans le cadre de notre travail présenté ici, focalisé sur les pratiques de différents acteurs.

En informatique, les analystes programmeurs se sont confrontés les premiers à la question complexe de délimiter les différentes phases dans le processus de production d'un logiciel, depuis sa conception jusqu'à sa commercialisation. En créant de logiciels pour répondre à des demandes et des besoins spécifiques d'un client, ils ont fait face à des difficultés d'appréhender les variations et les interdépendances entre les situations de conception, d'usage et celles de l'évaluation de l'utilisation du logiciel. Forts de leur expérience pratique et d'une demande croissante pour la création de nouveaux logiciels, ces spécialistes du développement informatique ont défini des méthodes, à travers des règles et des principes à respecter pour une production rapide et efficace de leurs produits. Ces méthodes se regroupent désormais sous une série de paradigmes méthodologiques en sciences informatiques comme celui des méthodes agiles (Leon et Koch, 2004), ou encore des méthodes centrées utilisateurs (Bainbridge, 2004) et celui des méthodes de recherche orientées par la conception (Barab et Squire, 2004). Tous ces paradigmes se caractérisent par une démarche expérimentale itérative (alternant des phases de conception avec des phases d'expérimentation et donc d'usage et d'évaluation) et incrémentale (améliorant les logiciels à la suite de chaque expérimentation).

Inspirés du succès de ces méthodes dans le développement de logiciels informatiques les vingt dernières années, les professionnels de la conception de jeux de formation numériques, connus principalement sous le nom de jeux sérieux (Abt, 1987), ont rapidement intégré et formalisé les principes pragmatiques de la réitération et de la 
démarche collaborative entre les différentes parties prenantes dans la conception des jeux (Plantec, 2010). De plus, parallèlement à ces avancées technologiques, le domaine de jeux sérieux a rapidement interrogé les champs d'applications possibles de la pédagogie numérique et l'intérêt d'élargir à la fois leurs objectifs d'apprentissage et les moyens mobilisés pour leur réalisation: d'un côté, il s'agit d'aller au-delà de l'acquisition de savoirs disciplinaires vers le développement d'autres compétences permettant à l'apprenant de mobiliser, d'associer et d'appliquer son savoir-faire dans la vie réelle (pour un exemple sur les interventions psychiatriques, voir Fleming et al., 2016 ; pour un exemple concernant la formation clinique des infirmiers voir Tan et al., 2017). De l'autre côté, il est question de ne pas se limiter à l'usage du seul outil numérique et d'intégrer différents types de supports pour la réalisation des tâches demandées dans le jeu. Influencés par des travaux comme ceux de David Shaffer sur les epistemic games (Shaffer, 2006) et le développement des méthodes de conception en informatique, des chercheurs français en sciences de l'éducation, en collaboration avec des enseignants du secondaire et des experts en formation professionnelle, ont proposé d'utiliser un nouveau vocable pour les jeux sérieux élargis dont il est question ici : les jeux épistémiques numériques (Sanchez et EminMartinez, 2014). C'est ce vocable qui a été retenu pour notre projet de recherche et que nous utiliserons également dans cet article pour désigner les jeux de formation hybrides.

Mais revenons aux trois activités représentant trois phases plus ou moins distinctes du processus de conception d'un jeu de formation: la segmentation du processus de production d'un jeu en différentes phases présente de toute évidence des avantages lors de la conception d'un jeu de formation numérique, notamment au niveau de l'identification de problèmes techniques ou encore au niveau de la conception d'un dispositif ajusté dans une perspective ergonomique. Pour autant, à notre connaissance, la littérature scientifique n'a pas encore documenté ni les échanges réels des acteurs impliqués au sein de ces différentes phases qui leur permettent d'obtenir un produit finalisé, ni la manière dont les «passages » entre les différentes phases influencent ces échanges. Or, les stratégies d'apprentissage des jeux de formation émergent au fil de ces multiples interactions entre concepteurs, joueurs et évaluateurs. C'est la raison pour laquelle l'analyse des caractéristiques interactionnelles de leurs échanges mérite une attention particulière. Par ailleurs, l'ouverture actuelle vers des environnements hybrides (numériques et non numériques) et des objectifs d'apprentissages plus larges complexifie la nature des interactions entre les acteurs durant les différentes phases de la conception et interroge à son tour les pratiques mobilisées.

La présente étude est issue du projet de recherche $\mathrm{JENlab}^{1}$ qui s'intéresse précisément à l'étude des pratiques et des ressources associées sous-jacentes à la production de jeux de formation hybrides alliant des supports numériques et non numériques.

À partir de la conception de deux jeux de ce type, expérimentés dans des classes de lycée et jusqu'à leur implémentation, le projet JENlab exploite un appareillage conceptuel pluridisciplinaire. Ce dernier combine des analyses de contenu avec des analyses des interactions, ou encore des analyses dans le temps et des études de cas. L'objectif est alors d'assurer la lisibilité des ingrédients dynamiques, de configurer autant de ressources différentes pendant et entre les séances de conception, et d'assurer des évaluations en classe. Au-delà de l'intérêt théorique que cela représente, la problématisation des ressources mobilisées est nécessaire à une meilleure compréhension des mécanismes mis en œuvre pour la production d'un jeu et pour l'apprentissage des joueurs. La compréhension de ces mécanismes permet alors de mieux saisir les conditions qui pourraient avoir un effet sur l'efficacité de ce type de dispositifs.

\footnotetext{
${ }^{1}$ Projet de recherche financé par l'Agence Nationale de la Recherche Scientifique, dirigé d'abord par E. Sanchez (Ifé de Lyon), remplacé par A. Serna (Liris, Lyon) depuis 2016: Programme Apprentissages 2013, référence ANR-13-APPR-0001.
} 
Dans cet article, fondé sur des enregistrements vidéo à la fois des séances de conception et d'implémentation pendant les deux premières itérations de la conception de deux jeux du projet JENlab, nous étudions qualitativement les processus interactionnels sous-jacents à leur conception. Le cadre de référence mobilisé pour cette étude et ses résultats s'ancrent dans des recherches variées qui s'intéressent à la construction sociale et collective des pratiques de conception appliquées aux jeux épistémiques numériques (Filliettaz, 2009; Shaffer, 2006; Widmer, 2001). Nous souhaitons alors montrer comment les différentes phases de la conception d'un jeu configurent les interactions et en quoi ce qui se passe en interaction permet de (re)définir à son tour ces phases du processus de la conception.

Dans un premier temps, nous définirons le dispositif de jeux épistémiques numériques avant de présenter les méthodes qualitatives et les outils d'analyse mobilisés. Ensuite, nous présenterons nos principaux résultats d'analyse répondant à la question centrale de cet article sur les ressources mobilisées pour la conception de jeux épistémiques numériques. Nous terminerons avec une synthèse des enseignements qui s'imposent et les perspectives auxquelles ouvre notre démarche de recherche.

\section{Les jeux épistémiques numériques : la définition actuelle}

Les jeux épistémiques numériques ou JEN représentent une alternative prometteuse aux jeux sérieux. Leurs traits caractéristiques (tels qu'ils ont été conçus dans le cadre du projet JENlab et des deux jeux étudiés) peuvent être décrits selon deux axes et comme suit :

- simulation de situations professionnelles «authentiques» avec des objectifs pédagogiques clairs et des résultats formulés au préalable en termes de formation et d'évaluation,

- mobilisation à la fois des technologies modernes (telles que la réalité mixte, les appareils mobiles et les plates-formes de collaboration) et des méthodes pédagogiques variées (par exemple les réunions en groupe, la tenue de cahiers de charges etc.).

Autrement dit, en proposant une palette de ressources distribuées dans des dispositifs éducatifs multi-supports, les JEN visent l'acquisition de compétences permettant aux apprenants de faire face à des problèmes qui n'ont pas de bonnes solutions à priori.

Au niveau des objectifs pédagogiques, ils se distinguent des jeux sérieux en ce qu'ils ne visent pas l'acquisition de savoirs disciplinaires dont l'appropriation pourrait être par exemple évaluée à travers des tests de vérification des connaissances. Au niveau technique, ils se distinguent également des jeux sérieux, puisqu'ils utilisent toute une variété de supports pédagogiques de nature variée comme les réunions, la tenue de cahiers des charges ou encore la réalisation d'enquêtes.

Les JEN sont censés offrir aux élèves la possibilité de se livrer à des expériences proches de celles qu'ils auront dans la vie active, c'est-à-dire des problèmes complexes, pouvant être traités de différentes manières. Ce type de jeux les amène à mettre en place des stratégies de résolution de problèmes. Le parti pris est de penser que ces stratégies seront remobilisées lors d'expériences professionnelles futures et dans ce sens qu'elles faciliteraient l'insertion professionnelle des apprenants (Shaffer, 2006).

\section{Méthodes et outils}

À l'aide d'une série d'enregistrements audiovisuels pendant les trois itérations de la conception des prototypes de deux JEN (environs 100h de données) nous avons pu observer dans le détail les pratiques de différents acteurs impliqués dans la conception et dans le jeu, les manières dont ils transformaient dans l'action des ingrédients de l'interaction en des ressources afin de pouvoir accomplir l'activité entreprise. 
Pour une meilleure description des phénomènes observés nous avons procédé à la rédaction des synopsis présentant des descriptions synthétiques des activités observées et/ou à des transcriptions plus ou moins fines des séquences repérées. En s'inspirant de la démarche de l'Analyse Conversationnelle issue de l'ethnométhodologie (Nguyen, 2012; Mondada, 2006) pour l'analyse des interactions, les transcriptions ont été réalisées de sorte à rendre compte des détails de l'interaction tels que l'intonation ou les chevauchements de tours de parole, et ceci à l'aide des conventions présentées en fin d'article. Nous nous sommes efforcées alors d'identifier dans nos données des occurrences de phénomènes de nature comparable.

Le contexte de travail pluridisciplinaire de notre projet impliquant des chercheurs en sciences de l'éducation, en sciences cognitives, en sciences du langage, en didactique, en informatique, des enseignants du secondaire et des professionnels de la conception de jeux de formation, fournit un appareillage essentiel pour des résultats optimaux dans une démarche de recherche applicative et appliquée (Lund, 2016). L'ensemble de ses orientations pluridisciplinaires (plus ou moins revendiquées comme appartenant à des paradigmes différents selon les acteurs) a mis en évidence, au fil des collaborations, des continuités possibles entre les différents domaines scientifiques et professionnels du projet. Il se trouve que ces domaines partagent des hypothèses épistémologiques, bien qu'elles soient explorées avec des cadres théoriques et méthodologiques différents. Ce sont ces convergences, délimitant notre cadre de référence, que nous allons présenter maintenant.

\subsection{Cadrage de la recherche}

Notre perspective repose sur deux approches avec des entrées complémentaires mais non exclusives qui s'enrichissent mutuellement : une approche interactionnelle et une approche sociocognitive. L'approche interactionnelle s'intéresse ici en priorité aux modalités de l'accomplissement situé de l'apprentissage. En se focalisant sur les pratiques concrètes à travers lesquelles les activités formatives sont menées, l'approche interactionnelle permet de rendre compte des pratiques ordonnées et systématiques par lesquelles les participants organisent ces activités (Filliettaz, 2012; Nguyen, 2012). Délimitées par des procédés langagiers verbaux et non verbaux parfois très subtils, l'analyse de ces pratiques fournit un éclairage nouveau sur des problématiques fondamentales pour la conception de jeux épistémiques numériques telles que la collaboration, la négociation, la transmission et l'évaluation. L'approche sociocognitive adoptée ici se fonde sur l'analyse des représentations sociales des apprenants et s'attelle à décrire une série des principes organisateurs de leur expérience professionnelle (Shaffer, 2006). À partir des situations concrètes, elle permet de généraliser les traits définitoires et les organisations invariantes de certaines classes de situations dans les activités étudiées et de faire des liens entre les procédés interactionnels mobilisés pour l'accomplissement de ces situations.

Dans cette double approche interactionnelle et sociocognitive, l'apprentissage en tant qu'activité cognitive est conceptualisé comme résultant de plusieurs activités situées que nous pouvons saisir à travers l'analyse fine des situations réelles. En effet, si les grains d'analyse de ces deux approches varient, toutes les deux s'accordent quant à l'importance de documenter les conditions situées dans lesquelles l'activité de l'enseignant et l'apprentissage des apprenants opèrent ensemble en interaction. Nous considérons ainsi que les modalités des interactions entre l'enseignant et les apprenants, selon l'espace matériel et les supports pédagogiques, peuvent soit faciliter soit limiter les opportunités d'apprentissage que le dispositif de formation offre à l'apprenant (Fuller et Unwin, 2003).

Puis, les deux approches, de concert, renvoient à l'analyse des pratiques langagières verbales et non verbales dans l'interaction (interactions entre participants; interactions homme-machine) comme un lieu prototypique des interactions sociales (Mondada, 2006). 
Enfin, l'analyse de la participation, telle qu'elle a été initialement définie par Goffman (1981), est considérée comme un outil puissant mettant en lumière les transformations dynamiques des catégorisations des participants lorsque nous étudions par exemple leur engagement dans le jeu, leur expertise et ainsi de suite (pour une étude récente sur la question de la participation dans le jeu voir Baldauf-Quilliatre et Colón de Carvajal, 2015 ou encore Tong et al., 2016). Ces transformations, une fois identifiées, sont considérées comme un indicateur à la fois du jeu des apprenants et d'un apprentissage en train de se faire, autrement dit d'une transformation de l'expertise des joueurs et de leurs catégories d'acteurs.

\subsection{Insectophagia et Rearth}

Dans le cadre du projet JENlab, deux prototypes de jeux épistémiques numériques adressés respectivement à des élèves de lycée général et des élèves de lycée technologique ont été conçus : le jeu Insectophagia et le jeu Rearth.

Insectophagia vise la création d'une entreprise qui élève des insectes comestibles, les transforme en des produits alimentaires et les commercialise.

Rearth met en scène la destruction imminente de la Terre. Ce jeu exige l'exploration de plusieurs planètes de l'Univers à l'aide d'une sonde qui se déplace de planète en planète et recueille de données géologiques dont l'étude permettrait aux joueurs de choisir une nouvelle planète habitable.

Respectivement aux méthodes agiles et aux méthodes centrées utilisateur, les deux jeux sont conçus de manière incrémentale en trois itérations. Ces itérations sont composées d'au moins deux séances de conception au début de l'année, suivies d'une évaluation en conditions écologiques dans plusieurs écoles partenaires du projet avant la fin de l'année scolaire.

\section{Nos principaux résultats}

Avant de commencer il est important de noter que dans nos données, nous avons constaté que l'activité de l'évaluation n'arrive que de manière artificielle en troisième position après la conception et l'usage. En réalité, l'évaluation est une activité interactionnelle permanente, un travail incessant entre les acteurs qui alimente et traverse à la fois les activités de conception et celles du jeu. Constitutive des séances de conception elle est au fondement de toutes les actions entreprises par les concepteurs (en amont ou en aval des séances d'expérimentation). Constitutive des séances des jeux, elle permet de rendre compte de l'engagement plus ou moins fort des joueurs, de la manière dont les uns se positionnent vis à vis du jeu et des autres joueurs, de la manière dont les joueurs s'approprient le jeu et ses objectifs ludo-pédagogiques.

La présentation des résultats ici est divisée selon les endroits physiques où ont eu lieu les enregistrements : les classes de lycée ou les séances de jeu et les salles de conception. Ces espaces séparés sont la "scène » d'activités complexes dont l'étude approfondie rend compte des ressources interactionnelles que les acteurs mobilisent pour accomplir les tâches qu'on leur a confiées : décider de la suite d'un jeu et jouer le jeu. 


\subsection{Les séances de conception : quelles ressources interactionnelles?}

\subsubsection{La méthode itérative comme ressource}

Le tableau qui suit doit être lu par colonne et de gauche à droite. Il illustre un cas fréquent dans l'ensemble de nos données, propre à la méthode itérative, consistant à apporter des modifications à l'organisation du jeu lors de la deuxième phase de conception et à la suite des premiers retours d'expérience en conditions écologiques.

Tableau 1. La réorganisation du rôle des enseignants.

\begin{tabular}{|c|l|l|}
\hline $\begin{array}{c}\text { Conception Phase } \\
\mathbf{1}\end{array}$ & $\begin{array}{c}\text { Jeu en classe } \\
\text { (première itération) }\end{array}$ & $\begin{array}{c}\text { Conception Phase } \\
\mathbf{2}\end{array}$ \\
\hline & $\begin{array}{l}\text { Constat : Interventions } \\
\text { «imprévues » de } \\
\text { l'enseignant, sollicitées } \\
\text { par les élèves sur des } \\
\text { savoirs disciplinaires }\end{array}$ & $\begin{array}{l}\text { Modifications: } \\
\begin{array}{l}\text { Préparation des } \\
\text { Rôle de en amont; } \\
\text { mise en place de } \\
\text { moments de } \\
\text { l'ébriefing }\end{array} \\
\text { animeignant le jeu }\end{array}$ \\
\end{tabular}

Lors de la première année, des séances de conception ex nihilo ont eu lieu réunissant les membres du projet. La réalisation de plusieurs ateliers a permis de fixer les objectifs pédagogiques des jeux à créer, de choisir les étapes importantes du scénario et les éléments ludiques et enfin de déterminer les rôles de différents acteurs dans le jeu.

Lors de ces séances, l'enseignant des classes ciblées par les nouveaux jeux s'est vu attribuer le rôle de l'animateur. Dans ce rôle il était censé valider les différentes étapes, distribuer les récompenses, surveiller la dynamique des groupes et ainsi de suite.

Lors de la première évaluation en situation de classe, nous avons pu observer grâce aux enregistrements vidéo que des interventions très fréquentes de la part de l'enseignant pendant les séances du jeu, sollicitées par les élèves, concernaient non pas directement le jeu mais des demandes de clarification et des explications au niveau des savoirs disciplinaires (tels que les équations mathématiques ou encore les unités de mesure), considérés comme acquis pour le bon déroulement du jeu.

Ces observations ont permis de mettre en évidence la nécessité de modifier le rôle de l'enseignant, allant du rôle d'un simple animateur à celui d'un véritable moniteur qui prépare les élèves en amont sur les thèmes disciplinaires abordés lors du jeu et les accompagne au fil du jeu avec l'organisation de séances de débriefing. Dans ce cas, c'est le principe de la réitération avec les observations, à la suite de la première expérimentation écologique, qui a permis aux concepteurs de juger de l'efficacité de la première organisation prévue et transformer le rôle et les tâches attribués à l'enseignant dans le jeu.

\subsubsection{Des procédés interactionnels comme ressource}

Les exemples qui suivent sont issus des séances de conception du jeu Insectophagia. Tous les noms des participants ont été anonymisés à l'aide de pseudonymes. Aurélie (AUR) et Lucas (LUC) sont les organisateurs des séances de conception et les animent. Michel (MIC), Alima (ALI) et Bernard (BER) sont des enseignants concepteurs.

Les exemples montrés ici illustrent deux catégories de pratiques retrouvées dans les séances de conception qui jouent un rôle important aux moments de négociations et de prise 
de décisions entre les participants. Il s'agit de séquences présentant deux formats récurrents et distincts : des séquences de propositions suivies d'une acceptation ou d'un refus et des séquences de répétitions ou de reformulations suivies d'une évaluation. Ces formats correspondent à deux procédés interactionnels récurrents par lesquels les participants transforment des propositions formulées par d'autres conduisant à la stabilisation de la conception d'un objet qui pourra alors être intégré ou pas dans le design final du jeu. Des séquences courtes ont été choisies ici mais il est important de noter que nous retrouvons les mêmes procédés interactionnels sur des séquences avec des interventions plus longues, parfois entrecoupées par d'autres séquences.

\section{Exemple 1 : Séquence de proposition/acceptation}

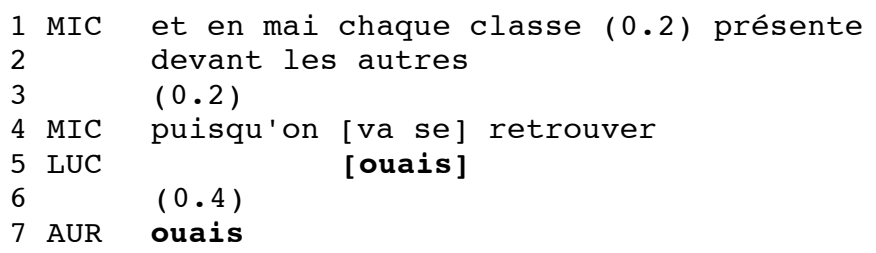

Dans cet extrait, Michel (MIC) propose que les élèves se retrouvent un mois après la fin de l'expérimentation écologique (lignes non montrées ici) pour présenter leur travail aux autres élèves. Sa proposition est accueillie favorablement par les animateurs des séances de conception, Lucas (LUC) et Aurélie (AUR). Les participants rendent ainsi visible le fait qu'ils sont d'accord et que la proposition est validée. En effet, une présentation en classe est organisée à la fin du jeu cette année-là.

\section{Exemple 2 : Séquence de proposition/ refus}

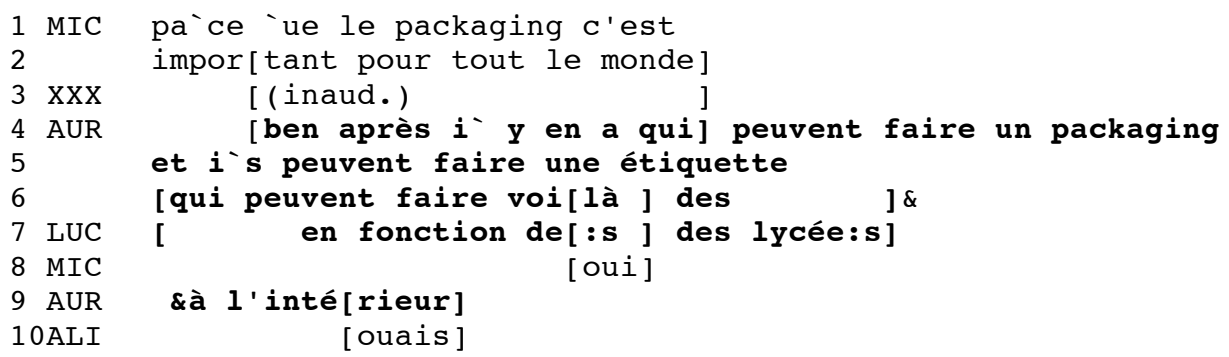

Dans cet extrait, Michel propose que les élèves s'engagent dans le jeu à produire un packaging pour les produits alimentaires à base d'insectes (lignes non montrées ici). Il finit sa proposition avec un énoncé évaluatif qui vient renforcer la proposition faite (1. 1-2). Les animateurs Aurélie et Lucas réagissent en formulant de manière collaborative une proposition alternative qui n'imposerait pas la création d'un packaging. Le packaging ne sera en effet pas imposé à tous les groupes qui participent à l'expérimentation.

\section{Exemple 3 : Séquence de reformulation/ évaluation}

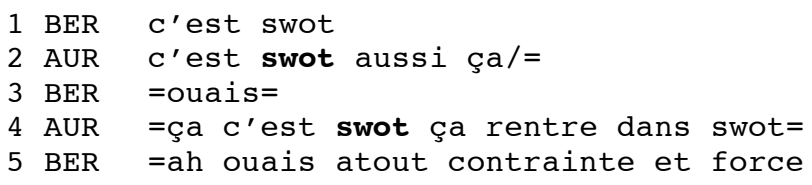




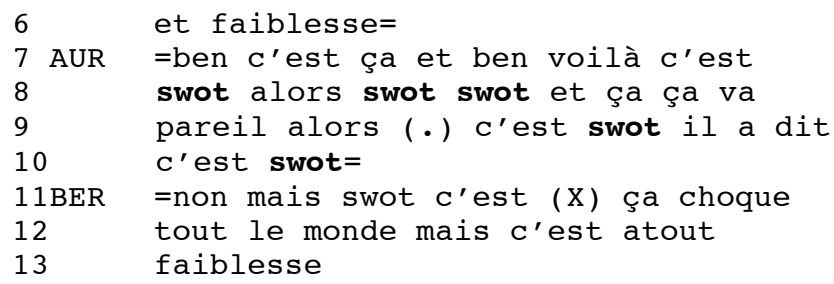

Dans cet extrait, nous observons la production de sept occurrences du mot « $\operatorname{swot}^{2} »$ de la part d'Aurélie, un terme introduit par Bernard à la ligne 1. SWOT renvoie à un modèle de planification des actions futures utilisé essentiellement dans le monde de l'entreprise. Les répétitions rapprochées de ce mot sont produites dans un énoncé qui n'apporte pas d'information supplémentaire, elles ouvrent la voie à des interprétations diverses concernant le positionnement d'Aurélie. Si nous ne savons pas ce que Aurélie pense, nous observons deux actions distinctes : Aurèlie interpelle Bernard lors de la septième répétition du mot swot («il a dit c'est swot», 9-10) à la suite de quoi Bernard produit une justification. Nous observons en effet la production d'une justification de Bernard aux lignes 11 à 13 qui rend visible le fait que les répétitions d'Aurélie constituent à ses yeux une critique de l'utilisation du mot swot («non mais swot c'est (x) ça choque tout le monde, 11-12) suivie d'une reformulation possible («mais c'est atout faiblesse », 12-13). Ce à quoi Aurélie ne réagit pas.

Saisir ces instants de conception en plein travail, au sein de séquences de négociation d'idées entre les participants permet de mettre en lumière les modalités par lesquelles s'organise l'activité de conception facilitant ou limitant l'introduction ou la continuation d'une proposition donnée. Dans ce sens, le caractère dynamique et indexical de l'interaction (Mondada, 2002) constitue une ressource à part entière que les participants exploitent pour accomplir les tâches prévues et prendre des décisions lors des phases de la conception. Des procédés interactionnels particuliers comme les séquences de propositions et les séquences de reformulation sont des instances concrètes dans le déploiement de cette ressource fondamentale, mobilisées pour parvenir à une situation finale avec l'accord (plus ou moins explicite) de tous.

\subsection{Les séances de jeu : quelles ressources interactionnelles ?}

\subsubsection{Les postures épistémiques comme ressource}

De manière générale, la posture épistémique se traduit dans des imputations et négociations des connaissances des participants et dans leur aptitude à répondre ou à réagir à certaines actions (Heritage, 2012). Différentes postures épistémiques sont mobilisées par les joueurs pour répondre aux attentes créées par les jeux et les enseignants. Deux champs d'expertise ont été identifiés dans nos données : (a) celui qui est directement lié à la thématique du jeu, traitant des questions de contenu, et (b) celui lié à des aspects techniques dépassant le cadre du jeu.

(a) Les postures épistémiques liées au thème du jeu

Trois exemples issus des séances de jeu Insectophagia documentent trois différentes postures épistémiques exhibant un engagement plus ou moins fort par rapport à

\footnotetext{
${ }^{2}$ Swot est un acronyme faisant référence à une méthode d'analyse utilisée au départ dans le cadre d'un plan marketing: S signifie strengths (forces), W weaknesses (faiblesses), O opportunities (opportunités), T threats (menaces).
} 
l'information donnée, ce qui se répercute sur le positionnement interactionnel du joueur vis à vis de ses collègues et peut entraîner des modifications quant à son pouvoir de décision ou encore de ses droits et obligations au niveau de la prise de parole et des opportunités à initier ou pas une nouvelle action. Mélanie (MEL), Laetitia (LAE), Ludivine (LUD) et Louise (LOU) sont des élèves qui jouent.

Exemple 4 : Lire une nouvelle information

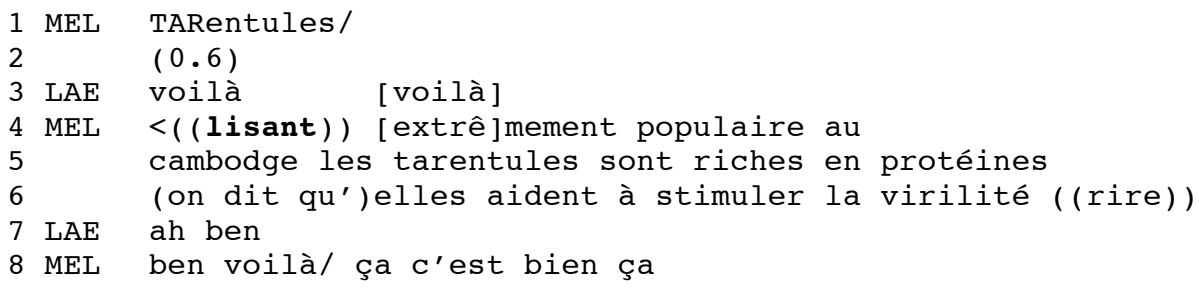

Dans cet extrait, le joueur Mélanie fait une recherche internet sur les apports de la consommation des tarentules (les joueurs doivent choisir un insecte à cultiver pour des produits comestibles à base d'insectes). Ce faisant elle rend visible qu'elle n'a pas une expertise particulière en la matière. De manière très fréquente dans nos données, des recherches sont réalisées par les joueurs sur internet ou dans d'autres documents écrits pour trouver des réponses à des questions qui émergent pendant la discussion. La lecture à haute voix, dans des séquences de recherche d'informations, manifeste l'importance de l'information trouvée qu'accorde le joueur pour le groupe et la suite du jeu.

Exemple 5 : Réitérer une déclaration/ opinion exprimée précédemment

1 LAE son goût est similaire au crabe/ tu vois c'est

2 pareil que $l^{\prime}$ crabe $j$ te $l^{\prime}$ 'avais dit

Comme illustré par cet exemple, les joueurs naviguent constamment entre ce qu'ils savent déjà et ce qu'ils ne savent pas. De manière intéressante, la référence à une discussion préalable confère aux joueurs qui la produisent une certaine expertise qu'ils peuvent potentiellement exploiter pour marquer une asymétrie avec leurs collègues comme dans l'exemple ci-dessus.

Exemple 6 : « Doing being » expert

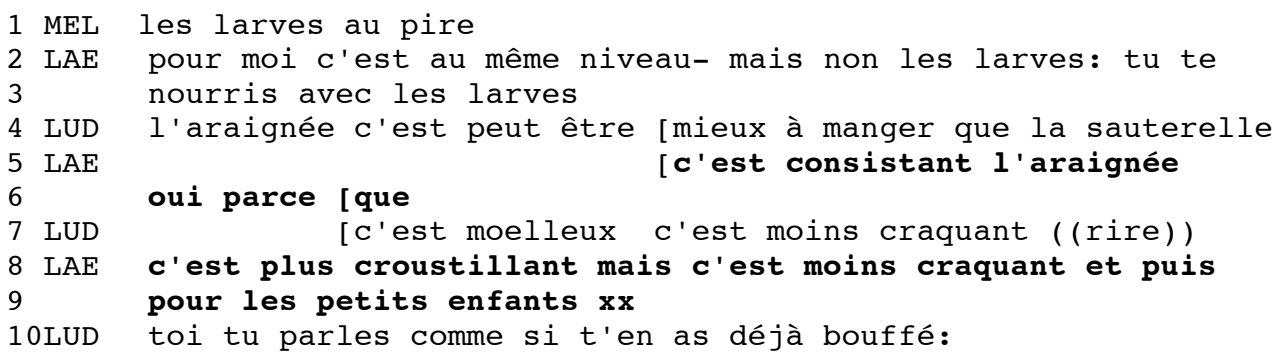

Ce troisième exemple en lien avec le thème du jeu est particulièrement intéressant. Il donne à voir des situations où le joueur s'engage pleinement dans son rôle, ce qui l'amène à défendre des points de vue qu'il ne défendrait pas en dehors du jeu. Ludivine le remarque bien en soulignant que Laetitia parle « comme si » elle en avait déjà mangé (ligne 10). 
(b) Les postures épistémiques liées aux aspects techniques du jeu

Exemple 7 : Se montrer expert dans d'autres domaines

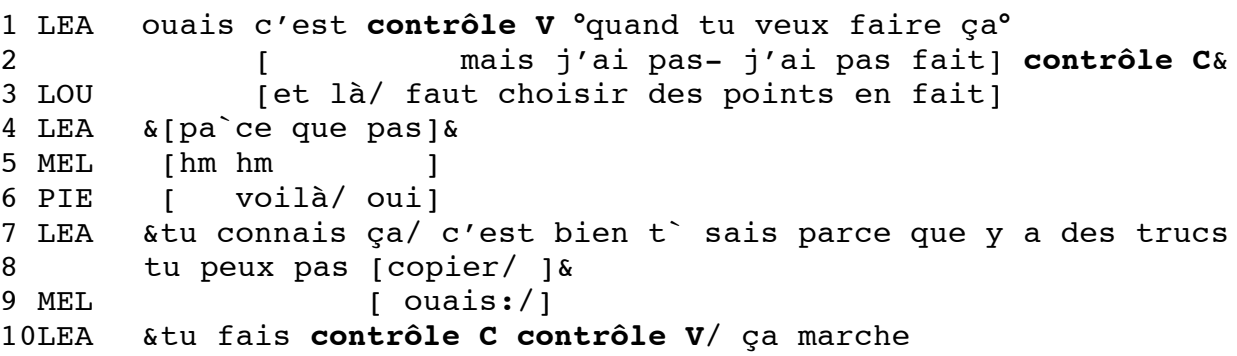

Ici, Laetitia donne des conseils à sa collègue concernant la copie de fichiers informatiques. Ce dernier exemple montre bien qu'en situation de formation les interactions s'organisent autour des expertises disponibles et des expertises à acquérir par les joueurs. Au-delà du cadre imposé par les dispositifs de formation, les joueurs mobiliseront d'autres ingrédients de l'interaction et créeront de nouvelles opportunités d'apprentissage pour euxmêmes et leurs collègues.

Si la conception du jeu rend légitime le développement de thématiques en lien avec le thème du jeu permettant aux joueurs d'afficher une certaine expertise plus ou moins forte sur un sujet particulier, nous avons vu que les participants mobilisent aussi d'autres savoirs leur permettant de créer des asymétries et d'introduire de nouveaux objets de savoirs qui ne sont pas prévus par le jeu. Cette question du lien configurant entre le jeu conçu et le jeu réalisé est particulièrement saillante au niveau des configurations de participation entre les joueurs. En effet, les modalités du jeu définissent les fonctions et les rôles que les participants peuvent endosser. Cependant lors du jeu les participants exploitent ce cadrage initial à d'autres fins pratiques. C'est ce que nous verrons dans la partie qui suit sur la mobilisation des configurations de participation comme ressource pour le jeu.

\subsubsection{Les configurations de participation comme ressource}

Nous avons vu que les JEN visent à opérer une transformation de l'expérience des joueurs en transmettant des savoirs disciplinaires mais aussi des manières d'agir dans une situation précise. L'introduction d'un jeu dans une classe est alors censée modifier le cadre dans lequel l'élève évolue habituellement et lui offrir de nouvelles opportunités d'apprentissage. Le jeu ne suffit pas à lui seul pour transformer l'environnement de l'apprentissage de l'élève mais dépend fortement des processus interactionnels qui émergent entre les élèves et entre les élèves et les enseignants. La question que nous nous posons alors est : comment pouvons-nous identifier ces transformations, comment savoir ce qui relève du jeu conçu et ce qui relève du jeu transformé par les actions situées des participants?

Dans nos données nous avons été interpellées par le fait que plusieurs séquences d'interaction contiennent des négociations faisant référence aux rôles préétablis des joueurs par les concepteurs. Les deux extraits qui suivent sont issus du corpus Rearth. Dans l'exemple 8, l'enseignant précise, suite à une question d'élève, que la durée d'installation sur une nouvelle planète dépend d'eux (sous-entendu des ressources géologiques disponibles) : 
Exemple 8 : Jeu de rôles, jeu d'énonciations

$\begin{array}{ll}01 & \text { JEA } \\ 02 & \\ 03 & \\ 04 & \text { MAT } \\ 05 & \\ 06 & \\ 07 & \\ 08 & \text { JEA } \\ 09 & \\ 10 & \text { CLA }\end{array}$

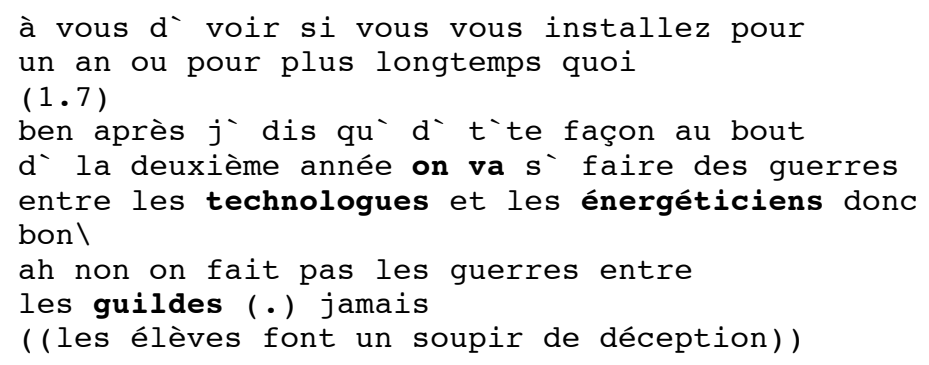

À la suite de la précision apportée par l'enseignant et d'une longue pause de 1,7 sec (1. 3), Matthieu manifeste son désaccord (1. 4-7). Nous observons qu'une référence explicite est faite aux rôles des jeux («entre les technologues et les énergéticiens », 1. 6) en utilisant le futur proche et le pronom personnel «on» («on va s'faire des guerres», 1. 5). De manière intéressante, le commentaire de Matthieu conduit à la transformation de la posture tenue par l'enseignant. En effet, l'enseignant apporte de nouvelles consignes (1.8) et par un jeu d'énonciation autour du terme " guildes » endosse de nouveau son rôle d'enseignant et recadre la classe («ah non on fait pas la guerre entre les guildes (.) jamais », 1. 8-9). L'annonce de l'absence de conflit entre les guildes dans le jeu provoque un certain mécontentement chez les autres élèves (1. 10).

Un peu plus tard, Matthieu montre des signes d'ennui. Ayant déjà lu la fiche de sa guilde chez lui, il doit attendre que le reste de la classe prenne connaissance des fiches. L'enseignant se rend compte de la situation et propose à Matthieu de lui envoyer une autre fiche (1. 1-4). La proposition de l'enseignant provoque une série de réactions auprès des élèves (rires, 1. 5) et le début d'un désaccord exprimé par Matthieu (1. 7).

Exemple 9 : Négociation des rôles

$\begin{array}{ll}01 & \text { JEA } \\ 02 & \\ 03 & \\ 04 & \\ 05 & \text { CLA } \\ 06 & \text { FAB } \\ 07 & \text { MAT } \\ 08 & \mathrm{KEV} \\ 09 & \mathrm{MAT} \\ 10 & \\ 11 & \mathrm{XXX} \\ 12 & \mathrm{MAT} \\ 13 & \mathrm{JEA} \\ 14 & \\ 15 & \\ 16 & \mathrm{MAT} \\ 17 & \\ 18 & \end{array}$

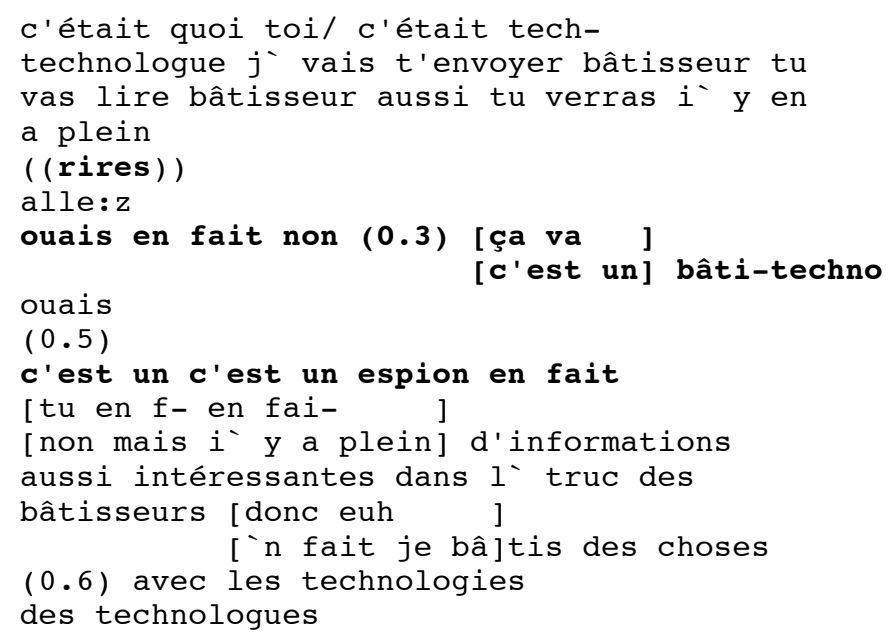

De manière intéressante, les élèves profitent de l'occasion pour intervenir en proposant de nouveaux rôles pour MAT (bâti-techno, espion) (1. 8-11) Les élèves exploitent le contexte du jeu pour intervenir et critiquer le statut de Matthieu dans le jeu $(1.8 ; 1.11)$. L'enseignant ne s'aligne ni avec les commentaires des élèves ni avec la tentative de Matthieu d'éviter la lecture d'une autre fiche (1. 13-15). Aussitôt Matthieu réagit et 
reformule son rôle de sorte à diminuer l'importance des informations contenues dans la fiche des bâtisseurs (1. 16-18).

Ces extraits sont particulièrement intéressants car ils illustrent une hybridité située des contextes de formation des JEN où les rôles des joueurs s'entremêlent à leurs statuts d'élèves, où les joueurs sur un ton taquin s'improvisent concepteurs, où l'enseignant doit s'ajuster aux contingences de la situation et proposer une nouvelle tâche à un élève qui s'ennuie, ce qui n'est pas initialement prévu dans le jeu. Les extraits montrent également que les transformations ne se font pas de manière lisse et linéaire mais de manière dynamique.

Ces aspects dynamiques du jeu, liés à l'activation de différentes expertises et à la transformation des configurations de la participation, ne sont habituellement pas considérés lors de la conception des jeux. L'ensemble de nos données et leur analyse nous invite à considérer la nécessité de s'éloigner des discours normatifs persistants, des clients, et de certains game-designers concernant l'évaluation du jeu et de l'engagement des joueurs en examinant les conditions locales d'accomplissement des activités.

\section{Remarques conclusives}

Pour mieux comprendre les problèmes pratiques que rencontrent les acteurs impliqués à la conception d'un JEN et les ressources qu'ils mobilisent pour accomplir les différentes tâches qui leur sont confiées, nous avons opté pour une double approche interactionnelle et sociocognitive, fondée sur des analyses détaillées des interactions. Le premier examen de $100 \mathrm{~h}$ de données audiovisuelles dont rend compte en partie cet article, nous a permis de dresser une liste systématique mais non exhaustive du type de ressources interactionnelles mobilisées, selon que l'on se trouve dans une séance de conception à proprement parler ou dans une séance d'expérimentation écologique du jeu en cours de conception. Les études de cas et l'étude longitudinale nous ont permis de rendre compte de la différence entre la participation conçue et celle qui est réalisée, des processus de catégorisation des participants plus ou moins explicites, de la manière dont les différents modules d'un jeu sont stabilisés lors des processus de conception, le tout étant susceptible de se transformer au gré des dynamiques interactionnelles des séances de conception et des séances de jeu.

Ainsi dans les séances de conception, au cours du cycle itératif, nous avons retrouvé la trace d'éléments qui se dégagent des séances des jeux, identifiés par les concepteurs et qui fonctionnent comme des points de repère à de nouveaux développements. Nous avons également mis en évidence deux procédés interactionnels spécifiques que l'on retrouve de manière systématique dans les séances de conception à chaque nouvelle proposition.

Concernant les séances d'expérimentation écologiques, deux phénomènes interactionnels ont été mis en évidence : les modifications du cadre de participation et l'émergence et la transformation des postures épistémiques. Dans les deux cas un rapport étroit est établi entre les situations de jeu et les variations de ces phénomènes. La manière dont le jeu est conçu et la manière dont il se déroule activent certaines thématiques et certaines catégories d'interactants. L'organisation prévue du jeu et son organisation émergente ont comme conséquence de contraindre la possibilité d'activer certaines autres catégories et thématiques.

Notre question de départ a été : «Quelles sont les ressources interactionnelles pour la conception des JEN? ». En étudiant les échanges de parole dans les séances de conception et les séances de jeu, nous avons montré que ce sont les dynamiques de ces interactions langagières qui sont finalement posées comme condition fondamentale des activités prévues avec les joueurs et donc des apprentissages. Nos analyses ont montré qu'aussi bien pour les séances de conception que pour les séances de jeu, l'ensemble des ingrédients de l'interaction (des représentations individuelles et sociales aux compétences 
interactionnelles) et les modalités de participation constituent des ressources privilégiées sur lesquels un concepteur averti peut agir et mieux accompagner les changements inhérents aux apprentissages non disciplinaires.

À partir d'une liste restreinte de phénomènes interactionnels ciblés, notre étude espère accompagner les concepteurs qui souhaitent entreprendre une démarche d'analyse des interactions en situation de jeu de formation. Cette liste peut bien évidemment être modifiée, mais en considérant les détails des interactions et en s'éloignant des discours prescriptifs et des modélisations hâtives. Ainsi, les concepteurs peuvent à travers l'analyse des pratiques réelles laisser une place pour la résolution des tensions qui existent entre les savoirs acquis et les savoirs à acquérir chez les joueurs ou encore celles qui subsistent au niveau des limitations techniques.

\section{Conventions de transcription}

[]

(.)

(2)

/

$\&$

(des)

((rit))

:

$\operatorname{xxx}$

extra

$=$

quo- chevauchement (début \& fin)

micro-pause $(<0,2$ secondes)

pause en secondes

intonation montante/ descendantel

continuation du tour de parole

essai de transcription

délimitation des phénomènes

allongement

segment incompréhensible

segment accentué

latching

troncation
${ }^{\circ}$ okay $^{\circ} \quad$ murmuré

NON saillance perceptuelle

>>okay<< accélération

\section{Bibliographie}

Abt C. C. (1987). Serious Games, Lanham: University Press of America.

Bainbridge W. S. (2004). Berkshire Encyclopedia of Human-Computer Interaction. Great Barrington: Berkshire Publishing.

Baldauf-Quilliatre H. \& Colón de Carvajal I. (2015). Is the avatar considered as a participant by the players? A conversational analysis of multi-player videogames interactions. PsychNology Journal, vol. 13, n² 2-3, p. 127-148.

Barab S. \& Squire K. (2004). Design-based research: Putting a stake in the ground. Journal of the Learning Sciences, vol. 13, n 1, p. 1-14.

Filliettaz L. (2009). Les dynamiques interactionnelles de l'accompagnement en formation professionnelle initiale: le cas de l'apprentissage sur la place de travail. Bulletin suisse de linguistique appliquée - VALS-ASLA, vol. 90, p. 37-58.

Filliettaz L. (2012). Affording learning environments in workplace contexts: an interactional and multimodal perspective. International Journal of Lifelong Education, vol. $32, \mathrm{n}^{\circ} 1$, p. 107-122.

Fleming T. M., de Beurs D., Khazaal Y., Gaggioli A, Riva G., Botella C., Baños R. M., Aschieri F., Bavin L. M., Kleiboer A., Merry S., Lau H. M., Riper H. (2016). Maximizing 
the Impact of e-Therapy and Serious Gaming: Time for a Paradigm Shift. Frontiers in Psychiatry, vol. 7, $\mathrm{n}^{\circ}$ 65. [doi:10.3389/fpsyt.2016.00065].

Fuller A. \& Unwin L. (2003). Learning as apprentices in the contemporary UK workplace: creating and managing expansive and restrictive participation. Journal of Education and Work, vol. 16, n 4, p. 407-426.

Goffman E. (1981). Forms of talk, Philadelphia: University of Pennsylvania Press.

Gueudet G. et Trouche L. (dir.) (2010). Ressources vives. Le travail documentaire des professeurs en mathématiques, Rennes : Presses Universitaires de Rennes.

Guichon N. (2006). Langues et TICE: méthodologie de conception multimédia, Paris : Ophrys.

Heritage J. (2012). Epistemics in action: Action formation and territories of knowledge. Research on Language and Social Interaction, vol. 45, $\mathrm{n}^{\circ}$ 1, p. 1-29.

Le Boterf G. (2011). Ingénierie et évaluation des compétences. Paris : Éditions Eyrolles.

Leon A. \& Koch A. S. (2004). Agile Software Development: Evaluating the Methods for Your Organization, Boston: Artech House.

Lund K. (2016). Modeling the Individual Within the Group: an Interdisciplinary Approach to Collaborative Knowledge Construction. Habilitation à Diriger des Recherches. Université Grenoble Alpes, France.

Mondada L. (2002). L'indexicalité de la référence dans l'interaction sociale : constructions discursives du “je" et de 1'“ici". Revista da ABRALIN, vol. 1, n 1, p. 127-169. [http://revistas.ufpr.br/abralin/article/view/52706].

Mondada L. (2006). Une approche praxéologique et cognitive de la relation à autrui : les défis interdisciplinaires de Bernard Conein. À propos de la parution de Bernard Conein 'Les sens sociaux. Trois essais de sociologie cognitive'. Langage et société, vol. 117, p. 143-152.

Nguyen H. T. (2011). Developing Interactional Competence: A Conversation-Analytic Study of Patient Consultations in Pharmacy, London: Palgrave Macmillan.

Plantec J. Y. (2010). Approche agile du développement de familles de jeux sérieux destinés au contrôle aérien. Génie logiciel, vol. 95, p. 6-12.

Sanchez E. et Emin-Martinez V. (2014). Ils jouent, mais quel jeu jouent-ils ? Du jeu sérieux au jeu épistémique. In Actes de la 9ème Conférence des Technologies de l'Information et de la Communication pour l'Enseignement (TICE 2014), p. 25-36. [http://t.co/VnW07ag1IV].

Shaffer D. W. (2006). How Computer Games Help Children Learn, London/New York: Palgrave Macmillan.

Tan A. J. Q., Lee C. C. S., Lin P. Y., Cooper S., Lau L. S. T., Chua W. L., Liaw S. Y. (2017). Designing and evaluating the effectiveness of a serious game for safe administration of blood transfusion: A randomized controlled trial. Nurse Education Today, vol. 55 , p. 38-44.

Tong L., Serna A., Pageaud S., George S. \& Tabard, A. (2016). It's Not How You Stand, It's How You Move: F-formations and Collaboration Dynamics in a Mobile Learning Game. In Proceedings of the 18th International Conference on Human-Computer Interaction with Mobile Devices and Services (MobileHCI '16). ACM, 6 septembre 2016, Florence (Italie), p. 318-329. 
Widmer J. (2001). Catégorisations, tours de parole et sociologie. In M. de Fornel, A. Ogien et L. Quéré (dir.) L'ethnométhodologie. Paris : La Découverte, p. 207-238. 